This item was submitted to Loughborough's Research Repository by the author.

Items in Figshare are protected by copyright, with all rights reserved, unless otherwise indicated.

\title{
Symmetry reduction of Fourier kernels
}

PLEASE CITE THE PUBLISHED VERSION

PUBLISHER

(C) Elsevier

LICENCE

CC BY-NC-ND 4.0

REPOSITORY RECORD

Samson, J.H., and G.A. Evans. 2019. "Symmetry Reduction of Fourier Kernels". figshare. https://hdl.handle.net/2134/2224. 


\title{
SYMMETRY REDUCTION OF FOURIER KERNELS
}

\author{
J H Samson \\ Department of Physics, Loughborough University, \\ Loughborough, Leics LE11 3TU, United Kingdom \\ (Electronic address: j.h.samson@lboro.ac.uk) \\ G A Evans \\ Department of Mathematical Sciences, de Montfort University, \\ Leicester LE1 9BH, United Kingdom \\ (Electronic address: gaevans@dmu.ac.uk)
}

May 20, 1998

\begin{abstract}
Fourier transforms of functions of several variables invariant under certain symmetry groups are studied, with particular reference to functions $f\left(\mathbf{x}_{1} \cdots \mathbf{x}_{N}\right)$ of $N$ three-component vectors invariant under rigid rotations. Here we use symmetry to enhance the efficiency of evaluation of the integrals. The Fourier transform can be written as an integral $F(k)=\int d \mu(x) K(k, x) f(x)$ over rotationally invariant quantities $x$. The kernel $K$, the average of $\exp \left(i \sum \mathbf{k}_{i} \cdot \mathbf{x}_{i}\right)$ over the rotation group $S O(3)$, is reduced to a single integral, $\int_{0}^{1} J_{0}\left(\frac{1}{2}\left(A_{x x}+A_{y y}\right) u\right) J_{0}\left(\frac{1}{2}\left(A_{x x}-A_{y y}\right)(1-u)\right) \exp \left(i A_{z z}(2 u-1)\right) d u$, a function of the eigenvalues of the dyadic $\mathrm{A}=\sum_{i=1}^{N} \mathbf{k}_{i} \mathbf{x}_{i}$. For $N=1$ the familiar Hankel transform is recovered. For $N=2$ the kernel reduces to a single integral of elementary functions, equal to the local spin-flip propagator in a one-dimensional tight-binding antiferromagnet. A variety of forms is given, and useful asymptotic forms are found in various limits. Recent numerical methods for the evaluation of irregular oscillatory integrals are applied to the kernel in the $N=2$ case.
\end{abstract}

Keywords: Fourier transform, Hankel transform, kernel

Mathematical Reviews subject classification: 65T99, 42B99, 81V99

Published in Journal of Computational Physics, 142, 109-122 (1998)

Copyright (C)1998 by Academic Press http://www.europe.idealibrary.com

\section{Introduction}

The symmetry of the integrand in multivariate Fourier transforms often allows reduction of the number of integrations, enhancing numerical efficiency. For example, the $d$-dimensional Fourier transform of a spherically symmetric function in $\mathbb{R}^{d}$ reduces to a one-dimensional Hankel transform[1]:

$$
\int d^{d} \mathbf{x} e^{i \mathbf{k} \cdot \mathbf{x}} f(x)=\int_{0}^{\infty} d x x^{d-1} K(k x) f(x)
$$

where the kernel $K(k x)$ is the average of $e^{i \mathbf{k} \cdot \mathbf{x}}$ over the unit hypersphere:

$$
K(k x)=\int_{S^{d-1}} d \Omega e^{i k x \cos \theta}=(2 \pi)^{d / 2}(k x)^{1-d / 2} J_{d / 2-1}(k x) .
$$

[We recognise this as the spherically symmetric term in the general solution of the $d$-dimensional Helmholtz equation $-\nabla^{2} K(\mathbf{x})=k^{2} K(\mathbf{x})$.] As the $d$-1-dimensional angular integral is known in closed form, we are left with a single radial integral over $x$. This is the process we wish to generalise; such generalisation does not seem to be well known in the literature.

The Fourier transforms considered here are of functions of $N d$-vectors,

$$
F(t \mathbf{k})=\int \prod_{i=1}^{N} d^{d} \mathbf{x}_{i} e^{i t \sum_{i=1}^{N} \mathbf{k}_{i} \cdot \mathbf{x}_{i}} f(\mathbf{x})
$$


where we write $\mathbf{k} \equiv\left\{\mathbf{k}_{i} \in \mathbb{R}^{d}, i=1 \ldots N\right\}$ and $\mathbf{x} \equiv\left\{\mathbf{x}_{i} \in \mathbb{R}^{d}, i=1 \ldots N\right\}$; the factor $t$ is introduced to aid later asymptotic analysis. Suppose $f(\mathbf{x})$ is invariant under a group $G$, which in the present work is the group $S O(d)$ of proper rigid rotations of the frame of $N$ vectors $\left\{\mathbf{x}_{i}\right\}$ in $\mathbb{R}^{d}$. Thus we have

$$
f\left(\left\{g \mathbf{x}_{i}\right\}\right)=f\left(\left\{\mathbf{x}_{i}\right\}\right) \forall g \in G
$$

and

$$
F\left(\left\{g \mathbf{k}_{i}\right\}\right)=F\left(\left\{\mathbf{k}_{i}\right\}\right) \forall g \in G .
$$

Let us separate $\mathbf{x}$ (hence also $\mathbf{k}$ ) into a set of "radial" or "shape" components $x \in M(k \in M)$, invariant under rotations, and a set of "angular" components $g \in G$. The group $G$ is $d(d-1) / 2$ dimensional; the shape space $M=\mathbb{R}^{N d} / G$ can be parameterised in a variety of ways[2]. We therefore wish to write the Fourier transform $(3)$ as

$$
F(k ; t)=\int_{x \in M} d \mu(x) K(k, x ; t) f(x)
$$

where the kernel

$$
K(k, x ; t)=\int_{g \in G} d \mu(g) e^{i t \sum_{i=1}^{N} \mathbf{k}_{i} \cdot g \mathbf{x}_{i}}
$$

generalises the Hankel kernel (2), with the measures $d \mu$ to be defined.

The integrals discussed here arose in an attempt to generalise the Wigner function of a single spin[3] to two or more coupled spins. However, other applications spring to mind, such as calculation of the momentum wave function $\phi\left(\mathbf{p}_{1}, \mathbf{p}_{2}\right)$ in a ${ }^{1} S_{0}$ state of the helium atom, given the real-space wave function $\psi\left(\mathbf{x}_{1}, \mathbf{x}_{2}\right)$ in Hylleraas-type[4] coordinates $\left\{\left|\mathbf{x}_{i}\right|,\left|\mathbf{x}_{i}-\mathbf{x}_{j}\right|\right\}$. Similar manipulations appear in the study of scattering of polarised light from orientationally disordered uniaxial ellipsoidal particles[5].

The kernel cannot in general be expressed in closed form in terms of elementary functions (or indeed any special functions known to the authors). Our aim is therefore to reduce it into a numerically tractable form. In three dimensions (with an arbitrary number $N$ of vectors) we reduce the triple integral (7) to a single integral (28) involving Bessel functions. With $N=2$ (as in the helium atom) the integral simplifies further to a single oscillatory integral of elementary functions, given in Eqs. (33-34). Recent numerical methods for irregular oscillatory integrals allow efficient evaluation of these forms.

\section{Analytical evaluation of kernel}

The general problem is to evaluate the integral (7). The exponent is linear in the rotation matrix $R_{\mu \nu}(g)$ :

$$
S(g) \equiv \sum_{i=1}^{N} \mathbf{k}_{i} \cdot\left(g \mathbf{x}_{i}\right)=\sum_{\mu=1}^{d} \sum_{\nu=1}^{d} R_{\mu \nu} A_{\nu \mu}
$$

where

$$
A_{\nu \mu}=\sum_{i=1}^{N}\left(\mathbf{k}_{i}\right)_{\mu}\left(\mathbf{x}_{i}\right)_{\nu}
$$

[For $N$ sufficiently large and suitably chosen vectors, any real $d \times d$ matrix A can be expressed as a dyadic of this form.] We shall henceforth assume summation over repeated Greek indices $\mu, \nu=1 \ldots d$ (labelling components) and Roman indices $i, j=1 \ldots N$ (labelling vectors).

There is some gauge freedom here[2]. We choose the initial relative orientation of the $\mathbf{x}$ and $\mathbf{k}$ frames to maximise $S$ :

$$
S_{+} \equiv \max _{g \in G} S(g)=S(e)=\mathbf{k}_{i} \cdot \mathbf{x}_{i}=\operatorname{Tr} \mathrm{A}
$$

where $e$ is the identity element of $G$. This defines a unique relative orientation almost everywhere in shape space; any degeneracies are of no relevance to the subsequent derivation. 


\section{1 $N$ vectors in three dimensions}

Having formulated the general case, we now specialise to $d=3$. The integration (7) is then over the three-dimensional manifold of $S O(3)$. There are two well-known parameterisations of this group: in terms of axis and angle of rotation, and in terms of the Euler angles. The latter leads to a more useful form, but we give both for completeness.

We first parameterise the group by the angles $\theta$ and $\phi$ (the axis of rotation $\mathbf{n}$ in spherical polar coordinates), and $\psi$, the angle of rotation about this axis, with $0 \leq \theta \leq \pi, 0 \leq \phi<2 \pi, 0 \leq \psi \leq \pi$. The normalised invariant measure [6] is

$$
d \mu(g)=\frac{1}{4 \pi^{2}} \sin \theta d \theta d \phi(1-\cos \psi) d \psi
$$

and the rotation matrix is

$$
R_{\mu \nu}(\theta, \phi, \psi)=n_{\mu} n_{\nu}+\left(\delta_{\mu \nu}-n_{\mu} n_{\nu}\right) \cos \psi-\epsilon_{\mu \nu \xi} n_{\xi} \sin \psi .
$$

The condition (10) for $d=3$ implies the "zero-torque" condition

$$
\mathbf{k}_{i} \times \mathbf{x}_{i}=\mathbf{0}
$$

which ensures that the coefficient of $\sin \psi$ in $S(g)$ vanishes. The matrix A (9) is therefore symmetric, its eigenvectors defining an orthogonal coordinate system. We shall order the eigenvalues as

$$
A_{z z} \leq A_{y y} \leq A_{x x}
$$

We now proceed to compute the kernel. The exponent is given by

$$
S(g)=w(\mathbf{n})+\left(S_{+}-w(\mathbf{n})\right) \cos \psi
$$

where

$$
w(\mathbf{n})=n_{\mu} n_{\nu} A_{\mu \nu}
$$

is a linear combination of $l=2$ spherical harmonics:

$$
\begin{aligned}
w(\mathbf{n}) & =A_{x x} \sin ^{2} \theta \cos ^{2} \phi+A_{y y} \sin ^{2} \theta \sin ^{2} \phi+A_{z z} \cos ^{2} \theta \\
& =A_{z z}+\left[\frac{1}{2}\left(A_{x x}+A_{y y}-2 A_{z z}\right)+\frac{1}{2}\left(A_{x x}-A_{y y}\right) \cos 2 \phi\right] \sin ^{2} \theta .
\end{aligned}
$$

The kernel (7) is

$$
K(k, x ; t)=\frac{1}{4 \pi} \int_{0}^{\pi} \sin \theta d \theta \int_{0}^{2 \pi} d \phi \kappa(t w(\mathbf{n})) .
$$

where the integrand $\kappa(t w(\mathbf{n}))$ is

$$
\begin{aligned}
\kappa(t w) & =\frac{1}{\pi} e^{i t w} \int_{0}^{\pi} e^{i t\left(S_{+}-w\right) \cos \psi}(1-\cos \psi) d \psi \\
& =e^{i t w}\left[J_{0}\left(t\left(S_{+}-w\right)\right)-i J_{1}\left(t\left(S_{+}-w\right)\right)\right] \\
& =\frac{-i}{t} \frac{\partial}{\partial w}\left[e^{i t w} J_{0}\left(t\left(S_{+}-w\right)\right)\right]
\end{aligned}
$$

We proceed by computing the density of $w$,

$$
\rho(W)=\frac{1}{4 \pi} \int_{0}^{\pi} \sin \theta d \theta \int_{0}^{2 \pi} d \phi \delta(W-w(\mathbf{n})),
$$

to reduce the kernel to a single integral over $W$. Integrating first over $\phi$ and then over $\cos \theta$, we obtain (in the non-degenerate case $A_{z z}<A_{y y}<A_{x x}$ )

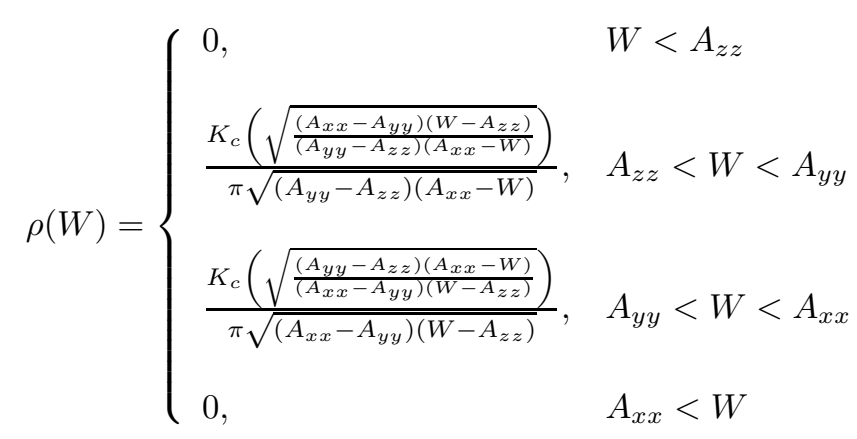


where $K_{c}$ is the complete elliptic integral of the first kind. This density has finite discontinuities at $W=A_{z z}$ and $W=A_{x x}$, and a logarithmic singularity at $W=A_{y y}$. Similar results for the density of other combinations of spherical harmonics have appeared elsewhere[5]. We obtain the kernel as

$$
K=\int_{A_{z z}}^{A_{x x}} \rho(w) \kappa(t w) d w .
$$

This integral is not yet in a form convenient for numerical integration.

We now obtain an equivalent result by integration over the Euler angles $0 \leq \alpha \leq 2 \pi, 0 \leq \beta \leq \pi .0 \leq$ $\gamma \leq 2 \pi$. The rotation matrix is[7]

$$
R=\left(\begin{array}{ccc}
\cos \beta \cos \alpha \cos \gamma & \cos \beta \sin \alpha \cos \gamma & -\sin \beta \cos \gamma \\
-\sin \alpha \sin \gamma & +\cos \alpha \sin \gamma & \\
-\cos \beta \cos \alpha \sin \gamma & -\cos \beta \sin \alpha \sin \gamma & \sin \beta \sin \gamma \\
-\sin \alpha \cos \gamma & +\cos \alpha \cos \gamma & \\
\sin \beta \cos \alpha & \sin \beta \sin \alpha & \cos \beta
\end{array}\right)
$$

and the normalised invariant measure is[2]

$$
d \mu(g)=\frac{1}{8 \pi^{2}} d \cos \beta d \alpha d \gamma
$$

The kernel is therefore

$$
K=\int_{g \in S O(3)} d \mu(g) e^{i t\left[\frac{1}{2}\left(A_{x x}+A_{y y}\right)(1+\cos \beta) \cos (\alpha+\gamma)+\frac{1}{2}\left(A_{x x}-A_{y y}\right)(-1+\cos \beta) \cos (\alpha-\gamma)+A_{z z} \cos \beta\right] .}
$$

Changing variables to $\alpha \pm \gamma$ and $u=(1+\cos \beta) / 2$ reduces the integral to

$$
K=\int_{0}^{1} J_{0}\left(t\left(A_{x x}+A_{y y}\right) u\right) J_{0}\left(t\left(A_{x x}-A_{y y}\right)(1-u)\right) e^{i t A_{z z}(2 u-1)} d u \text {. }
$$

Numerical tests confirm the equivalence of the integrals (24) and (28), although the latter is easier to evaluate. Expanding this kernel in a power series in $t$ gives

$$
\begin{aligned}
K=1 & -\frac{1}{6}\left(A_{x x}^{2}+A_{y y}^{2}+A_{z z}^{2}\right) t^{2} \\
& -\frac{i}{6} A_{x x} A_{y y} A_{z z} t^{3} \\
& +\frac{1}{120}\left[2\left(A_{x x}^{2}+A_{y y}^{2}+A_{z z}^{2}\right)^{2}-\left(A_{x x}^{4}+A_{y y}^{4}+A_{z z}^{4}\right)\right] t^{4}+O\left(t^{5}\right) .
\end{aligned}
$$

The real part of the kernel is invariant under the cubic symmetry group; the imaginary part changes sign under inversion.

\subsection{Two vectors in three dimensions}

We now further specialise to the case $d=3, N=2$. Figure 1 illustrates the rigid rotation of the pair $\left(g \mathbf{x}_{1}, g \mathbf{x}_{2}\right)$ with respect to the pair $\left(\mathbf{k}_{1}, \mathbf{k}_{2}\right)$, with $g \in S O(3)$. The torque-free condition (13) requires the four vectors $\left(\mathbf{k}_{1}, \mathbf{k}_{2}, \mathbf{x}_{1}, \mathbf{x}_{2}\right)$ to be coplanar, and the choice of axes (14) constrains them to lie in the $x y$ plane. The angle between $\mathbf{k}_{1}$ and $\mathbf{x}_{1}$ is

$$
\gamma=\arctan \left(\frac{k_{2} x_{2} \sin (\Theta-\chi)}{k_{1} x_{1}+k_{2} x_{2} \cos (\Theta-\chi)}\right)
$$

where $\chi$ is the angle between $\mathbf{x}_{1}$ and $\mathbf{x}_{2}$ and $\Theta$ the angle between $\mathbf{k}_{1}$ and $\mathbf{k}_{2}$ (with $0 \leq \chi \leq \pi$ and $0 \leq \Theta \leq \pi)$. The eigenvalues of $\mathrm{A}$ are then $A_{z z}=0, A_{y y}=\frac{1}{2}\left(S_{+}-S_{-}\right), A_{x x}=\frac{1}{2}\left(S_{+}+S_{-}\right)$, where we define

$$
\begin{aligned}
S_{ \pm} & =\sqrt{k_{1}^{2} x_{1}^{2}+k_{2}^{2} x_{2}^{2}+2 k_{1} k_{2} x_{1} x_{2} \cos (\Theta \mp \chi)} \\
& =\sqrt{k_{1}^{2} x_{1}^{2}+k_{2}^{2} x_{2}^{2}+2\left(\mathbf{k}_{1} \cdot \mathbf{k}_{2}\right)\left(\mathbf{x}_{1} \cdot \mathbf{x}_{2}\right) \pm 2\left(\mathbf{k}_{1} \times \mathbf{k}_{2}\right) \cdot\left(\mathbf{x}_{1} \times \mathbf{x}_{2}\right)}
\end{aligned}
$$




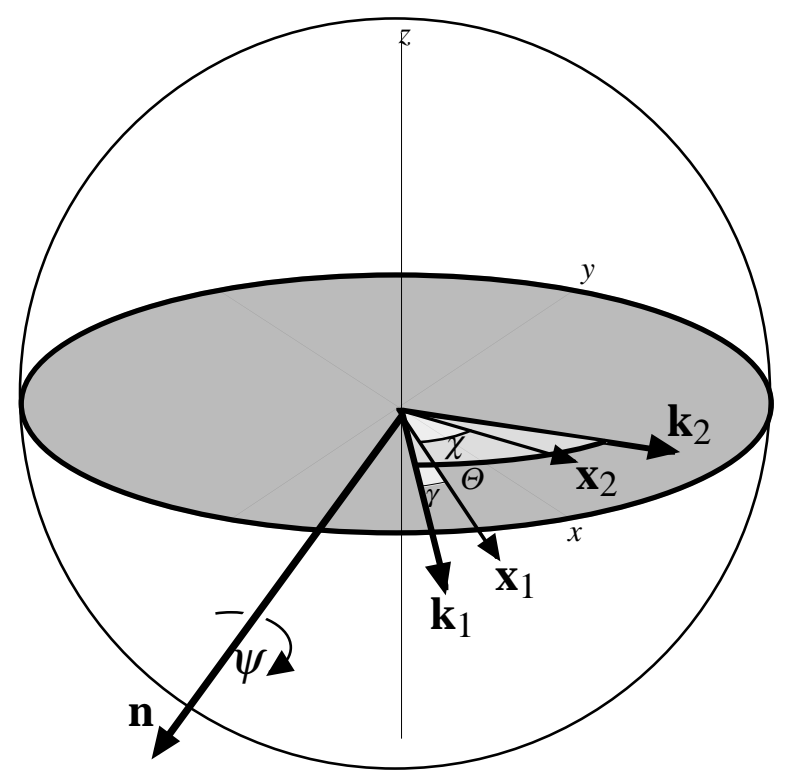

Figure 1: Geometry of integration for $d=3, N=2$

The definition (31) of $S_{+}$can easily be shown to agree with the earlier definition (10).

The integral (28) further simplifies in this case to a number of equivalent forms:

$$
\begin{aligned}
K\left(t S_{+}, t S_{-}\right)= & \int_{0}^{1} J_{0}\left(t S_{+} u\right) J_{0}\left(t S_{-}(1-u)\right) d u \\
= & \frac{2}{\pi t} \int_{S_{-}}^{S_{+}} \frac{\sin t z}{\sqrt{\left(S_{+}^{2}-z^{2}\right)\left(z^{2}-S_{-}^{2}\right)}} d z \\
= & \frac{2}{\pi} \int_{0}^{\pi / 2} \frac{\sin t \sqrt{S_{-}^{2}+\left(S_{+}^{2}-S_{-}^{2}\right) \sin ^{2} p}}{t \sqrt{S_{-}^{2}+\left(S_{+}^{2}-S_{-}^{2}\right) \sin ^{2} p}} d p \\
= & \int_{0}^{1} J_{0}\left(\frac{t\left(S_{+}+S_{-}\right) \sqrt{1-z^{2}}}{2}\right) J_{0}\left(\frac{t\left(S_{+}-S_{-}\right) \sqrt{1-z^{2}}}{2}\right) d z \\
= & \int_{0}^{1}\left[J_{0}\left(\frac{1}{2} t \sqrt{S_{+}^{2}-S_{-}^{2}} \sqrt{1-z^{2}}\right)\right]^{2} \cos \left(t S_{-} z\right) d z .
\end{aligned}
$$

[All but the first form, which comes from equation (28), were obtained by rotating $\mathbf{x}_{1}$ around $\mathbf{x}_{2}$, then rotating $\mathbf{x}_{2}$ around $\mathbf{k}_{1}$, and finally integrating over the angle between $\mathbf{x}_{2}$ and $\mathbf{k}_{1}$. The resulting integrals, functions of $k_{1} x_{1}, k_{2} x_{2}, \Theta$ and $\chi$, are then simplified by noting that they must depend only on the two variables $S_{-}$and $S_{+}$and using various standard results[8].] Numerical tests confirm that all these forms are indeed equivalent to each other and to the result (24) for $N=2\left(A_{z z}=0\right)$, although we have found no closed form expression in terms of known functions[8]. We note that these expressions are real. For $N>2$ the kernel is not in general real, as rotations in $S O(3)$ preserve the handedness of the frames. Figure 2 shows the form of the kernel for $N=2$.

\subsection{Tight-binding analogue}

We briefly note a connection between the integral (33) and one-electron propagators in a tight-binding system $[9]$. Consider a one-dimensional chain with nearest-neighbour hopping matrix element $\frac{1}{2} \sqrt{S_{+}^{2}-S_{-}^{2}}=$ 


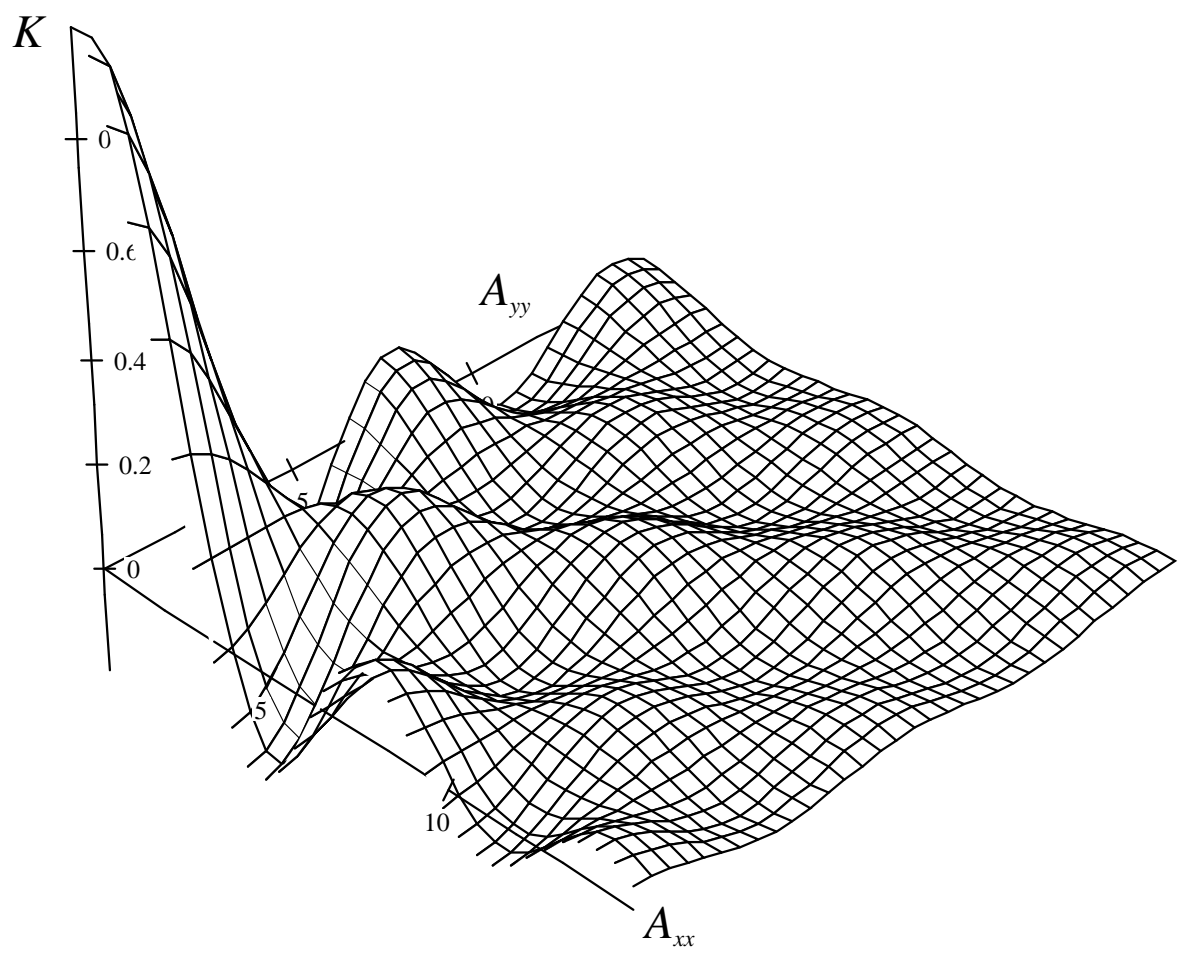

Figure 2: Kernel for $d=3, N=2$ as function of $A_{x x}$ and $A_{y y}$ with $t=1$.

$\sqrt{A_{x x} A_{y y}}$ and a staggered magnetic field $S_{-}=A_{x x}-A_{y y}$ :

$$
\begin{aligned}
H= & -\frac{1}{2} \sqrt{S_{+}^{2}-S_{-}^{2}} \sum_{i} \sum_{s=\uparrow}^{\downarrow}(|i s\rangle\langle(i+1) s|+| i s\rangle\langle(i-1) s|) \\
& -S_{-} \sum_{i}(-)^{i}(|i \uparrow\rangle\langle i \uparrow|-| i \downarrow\rangle\langle i \downarrow|)
\end{aligned}
$$

There are two bands, $-S_{+} \leq E \leq-S_{-}$and $S_{-} \leq E \leq S_{+}$, and the band structure is

$$
E_{ \pm}(p)= \pm \sqrt{S_{-}^{2}+\left(S_{+}^{2}-S_{-}^{2}\right) \sin ^{2} p}
$$

The local real-time propagator is defined as $G_{s s^{\prime}}(t)=-i \Theta(t)\left\langle 0 s\left|e^{-i H t}\right| 0 s^{\prime}\right\rangle$, the amplitude for an electron of spin $s^{\prime}$ on site 0 at time 0 to propagate to a spin $s$ state on site 0 at time $t$. The time average of the amplitude for an electron to remain on site 0 , and the spin-flip amplitude for an $x$-polarised electron, are both proportional to the kernel:

$$
\frac{1}{2 t} \int_{0}^{t} d t^{\prime}\left(G_{\uparrow \uparrow}\left(t^{\prime}\right)+G_{\downarrow \downarrow}\left(t^{\prime}\right)\right)=\frac{1}{2 \pi} \int_{-\pi}^{\pi} d p \frac{\sin E_{ \pm}(p) t}{E_{ \pm}(p) t}=K\left(t S_{+}, t S_{-}\right),
$$

and

$$
G_{\leftarrow \rightarrow}(t)=\frac{2}{\pi} \int_{S_{-}}^{S_{+}} \frac{S_{-} \sin E t d E}{\sqrt{\left(S_{+}^{2}-E^{2}\right)\left(E^{2}-S_{-}^{2}\right)}}=S_{-} t K\left(t S_{+}, t S_{-}\right) .
$$

(Compare Eqs. (34) and (33) respectively.) This may suggest other means of calculation of the kernel although the exact correspondence is not clear.

\subsection{Asymptotic analysis}

To understand the form of these oscillatory integrals, we investigate the leading large $t$ behaviour of the integrals $(33)$. In the general case $\left(t S_{+}-t S_{-} \gg 1, t S_{-} \gg 1\right)$, we use integral (33) and expand about the 
end-points:

$$
\sqrt{\left(S_{+}^{2}-z^{2}\right)\left(z^{2}-S_{-}^{2}\right)} \approx\left\{\begin{array}{cl}
\sqrt{\left(S_{+}^{2}-S_{-}^{2}\right) 2 S_{-} \xi}, & \xi=S_{-}+z \\
\text { or } \sqrt{\left(S_{+}^{2}-S_{-}^{2}\right) 2 S_{+} \xi}, & \xi=S_{+} z
\end{array} .\right.
$$

We can approximate the kernel by a sum of Fresnel integrals to give

$$
\begin{aligned}
K & =\frac{2}{\pi t \sqrt{S_{+}^{2}-S_{-}^{2}}}\left(\int_{0}^{\infty} \frac{\sin t\left(S_{-}+\xi\right) d \xi}{\sqrt{2 S_{-} \xi}}+\int_{0}^{\infty} \frac{\sin t\left(S_{+}-\xi\right) d \xi}{\sqrt{2 S_{+} \xi}}\right)+o\left(t^{-3 / 2}\right) \\
& =\sqrt{\frac{2}{\pi t^{3}\left(S_{+}^{2}-S_{-}^{2}\right)}}\left(\frac{\sin \left(t S_{-}+\pi / 4\right)}{\sqrt{S_{-}}}+\frac{\sin \left(t S_{+}-\pi / 4\right)}{\sqrt{S_{+}}}\right)+o\left(t^{-3 / 2}\right),
\end{aligned}
$$

i.e. $t^{-3 / 2}$ times the superposition of two oscillatory functions.

There are two degenerate cases, where the function is $O\left(t^{-1}\right)$ for large $t$. If the two $\mathbf{k}$ vectors (or the two $\mathbf{x}$ vectors) are nearly parallel, $t\left(S_{+}-S_{-}\right)=2 t A_{y y}$ will be small). In this case we find

$$
K \sim \frac{\sin t s}{t s} J_{0}\left(\frac{t\left(S_{+}^{2}-S_{-}^{2}\right)}{4 s}\right)-\frac{\left(S_{+}^{2}-S_{-}^{2}\right) \cos t s}{4 t s^{3}} J_{1}\left(\frac{t\left(S_{+}^{2}-S_{-}^{2}\right)}{4 s}\right)
$$

where $s=\sqrt{\left(S_{+}^{2}+S_{-}^{2}\right) / 2}$. We recover the $d=3$ Hankel kernel

$$
K=\frac{\sin t S_{+}}{t S_{+}}
$$

for $S_{-}=0$ (compare Eq. 2). In the other degenerate case both $k_{1} x_{1}=k_{2} x_{2}$ and $\Theta+\chi=\pi$ are satisfied, giving $S_{-}=0$ (or $\left.A_{y y}=A_{x x}\right)$. For small $S_{-}$we find

$$
K=\frac{J_{0}\left(t S_{-}\right)}{t \sqrt{S_{+}^{2}-S_{-}^{2}}}+\sqrt{\frac{2}{\pi t^{3} S_{+}^{3}}} \sin \left(t S_{+}-\pi / 4\right)+o\left(t^{-3 / 2}\right) .
$$

Inspection of the form (35) shows that the kernel is everywhere positive for $S_{-}=0$. Figure 3 shows the asymptotic forms for $t S_{+} \leq 20$. As expected, the generic form $\left(42\right.$, thin line) is accurate for $S_{+}=2 S_{-}$, while the other forms (45) and (43) are better approximations for small and large $S_{-}$respectively.

\section{$3 \quad$ Numerical studies}

We will concentrate here on the $N=2$ kernel. The versions of $K$ in equations (32-36) each exhibit different numerical difficulties which also depend on the values of the parameters involved. Conventional quadrature methods may be used on smooth integrands with rapid convergence [10] and such methods are typified by the classical Gauss-Legendre method and the method of Clenshaw and Curtis[11]. These methods fail to deliver such rapidly converging results in two major sub-cases which are the subjects of considerable literature. These are the cases in which the integrand has singularities in the range of integration, and when the integrand has a highly oscillatory part. This latter case is further sub-divided into regular oscillatory integrands typified by kernels of the form $\sin \omega x$ for large $\omega$, and irregular cases which have kernels such as $\sin \omega q(x)$. These cases are further extended if the oscillatory part is nontrigonometric such as a Bessel oscillation. All these cases arise in computing $K$. High order methods for the irregular oscillatory problem have only recently been set up [12].

Hence to return to computing $K$, consider first version (33). For this example the problem lies in the end point singularities which, if ignored by the blind application of the open Gauss-Legendre rule, will yield very slow convergence with around three figure accuracy for around 2000 integration points for typical values of the parameters. The easiest technique to employ here is to split the range of integration into two to yield two integrals each with singularities at zero. An application of the polynomial transformations of Evans, Forbes and Hyslop[13] with a simple quadratic transformation will give 14 correct digits with just a 16 point Clenshaw-Curtis rule. Hence the integral splits into

$$
K=K_{1}+K_{2},
$$


representing the lower and upper halves of the range of integration. Applying the singularity-removing transformations $z=S_{-}+\omega^{2} v^{2}$ and $z=S_{+}-\omega^{2} v^{2}$ respectively to each half-range, where $\omega^{2}=\left(S_{+}-S_{-}\right) / 2$, gives

$$
K_{1}=\frac{4 \omega}{\pi t} \int_{0}^{1} \frac{\sin t\left(\omega^{2} v^{2}+S_{-}\right)}{\sqrt{\left(\omega^{2} v^{2}+2 S_{-}\right)\left(S_{+}^{2}-\left(\omega^{2} v^{2}+S_{-}\right)^{2}\right)}} d v
$$

and

$$
K_{2}=\frac{4 \omega}{\pi t} \int_{0}^{1} \frac{\sin t\left(S_{+}-\omega^{2} v^{2}\right)}{\sqrt{\left(2 S_{+}-\omega^{2} v^{2}\right)\left(\left(S_{+}-\omega^{2} v^{2}\right)^{2}-S_{-}^{2}\right)}} d v .
$$

In the second case, the integral in (28) is an example of an irregularly oscillatory integrand, and a powerful approach to this is the direct application of the method of Evans and Webster[12]. The principle behind this method is to consider a rule of the form

$$
\int_{a}^{b} f(x) e^{i q(x)} d x=\sum_{i=1}^{M} A_{i} f\left(x_{i}\right)+E
$$

where

$$
x_{i}=\frac{b-a}{2} \cos \frac{\pi i}{M}+\frac{b+a}{2},
$$

and force this rule to be exact by setting $E=0$ for the set of functions given by $f_{j}(x)=p_{j}^{\prime}(x)+i p_{j}(x) q(x)$, where $p_{j}(x)=T_{j}(x), j=0, \ldots, M-1$ is the most effective choice for $p_{j}, T_{j}(x)$ being the $j$ th Chebyshev polynomial. For such a choice, (49) yields a set of $2 M$ linear algebraic equations for the real and imaginary part as the integral on the left hand side of (49) can be integrated exactly to give $\left.p_{j}(x) e^{i q(x)}\right|_{a} ^{b}$. Though the linear equations are ill-conditioned, the resulting computed weights can be shown to yield accurate quadrature rules to high accuracy as the residues of the defining linear equations are zero to machine accuracy. As in the previous case 14 significant digits are generated in 16 integration points.

The integral (35) is not so easy in that it is a product of two Bessel functions. The method of Evans and Webster has been extended to deal with kernels of the form $J_{n}(\omega q(x))$ but not with products of this type. The final case, namely equation (36), also exhibits a combination of oscillatory terms, and again the above comments apply.

\subsection{Numerical Results}

Practical tests were carried out on the four versions of the integral $K$ on a range of values of the defining parameters. For the first set of tests the value of $S_{+}$was fixed at 1.0, and $S_{-}$was allowed to take the values $0.01,0.5$ and 0.99 , and the scaling factor $t$ the values $0.5,1.0,5.0,10.0$ and 15.0. This range allows conventional Clenshaw-Curtis quadrature to be used, effectively taking no account of any of the oscillatory factors. This test establishes the equivalence of the four versions of the integral. The form (33) was integrated in two parts as in (47) and (48) to account for the singularity, but otherwise the integrals were performed directly.

The values obtained at convergence are shown in Table $\mathrm{i}$ and the number of function evaluations to achieve 14 figure accuracy for each of the methods is shown in Table ii, in the order of integrals (33-36).

\begin{tabular}{|c|c|c|c|}
\hline & \multicolumn{3}{|c|}{$S_{-}$} \\
\hline$t$ & 0.01 & 0.5 & 0.99 \\
\hline 0.5 & 0.97935894292357 & 0.97419724283520 & 0.95925546405373 \\
1.0 & 0.91972228144312 & 0.89960076847233 & 0.84297045100255 \\
5.0 & 0.14294005363476 & -0.081086970535548 & -0.19408619166121 \\
10.0 & 0.10645420869444 & -0.011174516638095 & -0.050355842266123 \\
15.0 & 0.07997198470926 & 0.035996484912053 & 0.04720017053884 \\
\hline
\end{tabular}

Table i: Numerical values of the kernel. See figure 3

Hence for moderate values of the defining parameters all four methods take a modest number of quadrature points, where we remember that two integrals are evaluated in the case of equation (33) for 


\begin{tabular}{|c|c|c|c|}
\hline & \multicolumn{3}{|c|}{$S_{-}$} \\
\hline$t$ & 0.01 & 0.5 & 0.99 \\
\hline 0.5 & $32,16,8,8$ & $16,16,8,8$ & $8,8,8,8$ \\
1.0 & $32,16,8,8$ & $16,16,8,8$ & $8,8,8,8$ \\
5.0 & $16,32,16,16$ & $8,16,16,16$ & $4,16,16,16$ \\
10.0 & $16,32,32,32$ & $8,32,32,32$ & $4,16,32,32$ \\
15.0 & $16,32,32,32$ & $8,32,32,32$ & $4,16,32,32$ \\
\hline
\end{tabular}

Table ii: Number $M$ of function evaluations required to obtain 14-digit accuracy with integrals (33-36).

each value. It is also beginning to appear that the cases (34-36) require increasingly large point numbers as $t$ increases and the integrands become more oscillatory. The method will become progressively more untenable as the oscillatory factor $t$ increases. In these regions the special oscillatory methods are used as illustrated in the next subsection in which the asymptotic formulae are investigated.

The reverse appears to occur for version (33). For this integral the oscillatory factor is $t \omega^{2}$ as in equations (47-48) and hence this factor increases for large $t$, but decreases as $S_{+}$approaches $S_{-}$which happens in the right hand column of Table i. Hence again in general, the direct integration of the full integrand with the singularity suppressed is only viable for non-large $t$ or for $S_{+}$close to $S_{-}$.

\subsection{Asymptotic regions}

It is of considerable interest to investigate how the asymptotic forms for $K$ behave numerically in the various asymptotic regions. The first region is for $t$ large and comparison may be made with the asymptotic result (42). It is clear that forms (35) and (36) will not be satisfactory with increasingly oscillatory terms. However cases (33) and (34) can be evaluated for large $t$ using the Evans and Webster algorithm, as long as the singularity in (33) is suppressed as in (47) and (48). This method holds its accuracy as the oscillatory factor increases for the same non-oscillatory part.

Table iii shows these results with $t$ taken as large as $10^{4}$, for $S_{+}=1$ and $S_{-}=0.5$.

\begin{tabular}{|c|c|c|c|c|}
\hline$t$ & $\begin{array}{c}M \text { for } \\
(34)\end{array}$ & $\begin{array}{c}M \text { for } \\
(33)\end{array}$ & $\begin{array}{c}\text { quadrature } \\
\text { value }\end{array}$ & $\begin{array}{c}\text { value } \\
\text { by }(42)\end{array}$ \\
\hline 10 & 32 & 16 & $-1.1174516638095(-2)$ & $-1.359508816(-2)$ \\
500 & 128 & 64 & $-3.573191319899(-5)$ & $-3.58733172(-5)$ \\
1000 & 128 & 64 & $-3.395355921834(-5)$ & $-3.3929922(-5)$ \\
5000 & 128 & 128 & $-1.81929623227(-6)$ & $-1.819600724(-6)$ \\
10000 & 128 & 128 & $-3.4645654920(-7)$ & $-3.46530191(-7)$ \\
\hline
\end{tabular}

Table iii: Number $M$ of function evaluations required, exact value of the kernel and asymptotic form for $S_{-}=0.5, S_{+}=1$.

For these values, the direct application of Clenshaw-Curtis to the full oscillatory integrand yields only 6 correct figures for 256 integration points at $t=1000$, and none at all at $t=10000$.

A new effect arises for these values, as the attainable accuracy will be below machine accuracy as the final values become smaller. This is a normal problem with oscillatory integrals where the integrand involves values of say order unity and the integral is say of order $10^{-n}$. Then inevitably the process must involve subtractive cancellation with the loss of $n$ digits. This effect can be observed in the evaluation of $\sin x$ itself for large arguments. As these high $t$ values will be used with other smaller $t$ results in the outer integrals, it is only the absolute errors which will be relevant, and these are sufficiently small not to cause a problem.

It is clear from Table iii that full attainable accuracy is achieved using both versions again remembering that two integrals are involved in case (33). Note that in both cases the increasing parameter $t$ is not just an oscillatory factor but also causes the non-oscillatory part of the integrand to become much less smooth, so requiring a higher point number. There is a balance here between the requirement for less absolute accuracy as $t$ increases and the effect of large $t$ reducing the smoothness of the non-oscillatory part of the integrand, so generating less accuracy. Hence it appears that either version could be used successfully for the numerical evaluation of $K$ throughout the required range. The computations were not 
pursued beyond $M=128$ as the associated overheads of the method become significant, being primarily caused by the solution of $2 M$ simultaneous equations.

The second asymptotic test involved using formula (43) for the case when $S_{+}$and $S_{-}$are close together. For this example, $t$ was taken as unity and $S_{-}$was ranged from 0.9 to 0.999 with $S_{+}=1$. These results are shown in Table iv.

\begin{tabular}{|c|c|c|c|c|}
\hline$S_{-}$ & $\begin{array}{c}M \text { for } \\
(34)\end{array}$ & $\begin{array}{c}M \text { for } \\
(33)\end{array}$ & $\begin{array}{c}\text { quadrature } \\
\text { value }\end{array}$ & $\begin{array}{c}\text { value } \\
\text { by }(43)\end{array}$ \\
\hline 0.9 & 64 & 32 & 0.8558818751252 & 0.856112785 \\
0.95 & 128 & 64 & 0.8488396689284 & 0.84888201 \\
0.99 & 128 & 64 & 0.84297045100255 & 0.842971673 \\
0.995 & 64 & 128 & 0.84222231359635 & 0.84222260464 \\
0.999 & $>128$ & 64 & 0.84162150547562 & 0.84162151666 \\
\hline
\end{tabular}

Table iv: Number $M$ of function evaluations required, exact value of the kernel and asymptotic form for $t=1, S_{+}=1$.

In Table iv the numerical method is seen to agree with the asymptotic estimates to greater and greater accuracy as the value of $S_{-}$approaches $S_{+}$. Because of this proximity, the numerical integrations can be achieved using Clenshaw-Curtis across the whole set of parameters in 32 integration points. The test was repeated for $t=10$ and $t=100$, and as $t$ increases the accuracy of the asymptotic estimate falls off as the crucial factor to be kept small is $t\left(S_{+}-S_{-}\right)$.

The third asymptotic formula is (45), and for this test, $S_{+}$and $t$ are fixed at 1 and 1000 respectively, and small values of $S_{-}$are used. These comparisons appear in Table v. The use of integral (34) in this regime proves increasingly difficult as the non-oscillatory part of the integrand approaches a root singularity as $S_{-} \rightarrow 0$.

\begin{tabular}{|c|c|c|c|}
\hline$S_{-}$ & $\begin{array}{c}M \text { for } \\
(33)\end{array}$ & $\begin{array}{c}\text { quadrature } \\
\text { value }\end{array}$ & $\begin{array}{c}\text { value } \\
\text { by }(45)\end{array}$ \\
\hline 0.01 & 64 & $-2.4124452500934(-4)$ & $-2.41229045676(-4)$ \\
0.005 & 64 & $-1.7289459328368(-4)$ & $-1.72879974829(-4)$ \\
0.001 & 64 & $7.6990137203799(-4)$ & $7.69917085643(-4)$ \\
0.0005 & 128 & $9.431733851343(-4)$ & $9.43188941036(-4)$ \\
0.0001 & 128 & $1.002205085(-3)$ & $1.00222058354(-3)$ \\
\hline
\end{tabular}

Table v: Number $M$ of function evaluations required, exact value of the kernel and asumptotic form for $t=1000, S_{+}=1$

\subsection{Numerical conclusions}

In the region for which $S_{+}, S_{-}$and $t$ all have modulus less then say 20, the direct application of ClenshawCurtis to the full integrand proves accurate and efficient on either of the four possible integrands. Versions (33) or (34) are preferred as this avoids the evaluation of Bessel functions. This region may be extended into large $t$ as long as $t\left(S_{+}-S_{-}\right)$remains small as in Table iv.

As soon as large values of $t$ or $S_{+}$occur then either version (34), or (33) with the singularities suppressed, are required and the irregular integrator of Evans and Webster becomes the effective tool. If in addition $S_{-}$is small then the integrand in (34) is closing in on a singularity and only (33) will be effective. An alternative is to use Clenshaw-Curtis on the range near to zero (say from 0 to $10 \pi /\left(S_{+} t\right)$ ) and Evans and Webster to complete the range to $\pi / 2$. This device then combines the sin function and the denominator to remove the singularity. Using these regions, it is possible to compute $K$ throughout its range.

\section{Conclusions}

The results obtained here, which we believe to be novel, provide a general framework for Fourier transforms of functions of several variables with symmetry. For $N$ vectors in three dimensions the rotational 
average of the exponential $e^{i \mathbf{k}_{i} \cdot \mathbf{x}_{i}}$ is given by the kernel (28). We have concentrated on the specific case of functions of two three-dimensional vectors, invariant under rigid rotations of the triangle defined by the vectors. Eqs. (32-36) list a number of forms for the kernel; different forms are appropriate in different parameter regions. For large values of the parameter $t$, the integrands are highly oscillatory, although accurate asymptotic expressions exist in the appropriate regions. Similar numerical techniques might be applicable to the general form of the kernel (28) for $N$ three-dimensional vectors, although we have not succeeded in reducing this to an integral of elementary functions. Finally, we note that although we have only reduced the number of integrations from $3 N$ to $3 N-2$, the oscillatory kernel for large wave numbers $t k$ falls off as $O\left(t^{-3 / 2}\right)$ in almost all directions, allowing faster convergence.

\section{Acknowledgments}

We are grateful to Professor Sasha Veselov for helpful discussions.

\section{References}

[1] G. B. Folland, Fourier Analysis and its Applications, p 247 (Wadsworth, Pacific Grove, 1992).

[2] R. G. Littlejohn and M. Reinisch, Gauge fields in the separation of rotations and internal motions in the $n$-body problem, Rev. Mod. Phys. 69, 213-275 (1997).

[3] J. H. Samson, Classical effective Hamiltonians, Wigner functions, and the sign problem, Phys. Rev. B51, 223-233 (1995).

[4] E. A. Hylleraas, Neue Berechnung der Energie des Heliums im Grundzustande, sowie des tiefsten Terms von Ortho-Helium, Z. Phys. 54, 347-366 (1929).

[5] A. P. Bates, K. I. Hopcraft and E. Jakeman, Particle shape determination from polarization fluctuations of scattered radiation, J. Opt. Soc. Amer. A 14, 3372 (1997).

[6] See, for example, H. F. Jones, Groups, Representations and Physics, p 243 (Adam Hilger, Bristol, 1990).

[7] See, for example, H. Goldstein Classical Mechanics, p 108 (Addison Wesley, Reading, 1950).

[8] I. S. Gradshteyn and I. M. Ryzhik, Tables of Integrals, Series and Products (Academic, New York, 1980).

[9] See, for example, E. N. Economou, Green's Functions in Quantum Physics (Springer, Berlin 1983).

[10] G. A. Evans, Practical Numerical Integration, (John Wiley and Sons, Chichester, 1993).

[11] C. W. Clenshaw and A. R. Curtis, A method for numerical integration on an automatic computer, Num. Math. 12 197-205, (1960).

[12] G. A. Evans and J. R. Webster, A high order progressive method for the evaluation of irregular oscillatory integrals, App. Num. Math. 23, 205-218, (1997).

[13] G. A. Evans, R. C. Forbes, and J. Hyslop, Polynomial transformations for singular integrals Int. J. Comp. Math. 14, 157-170, (1983). 

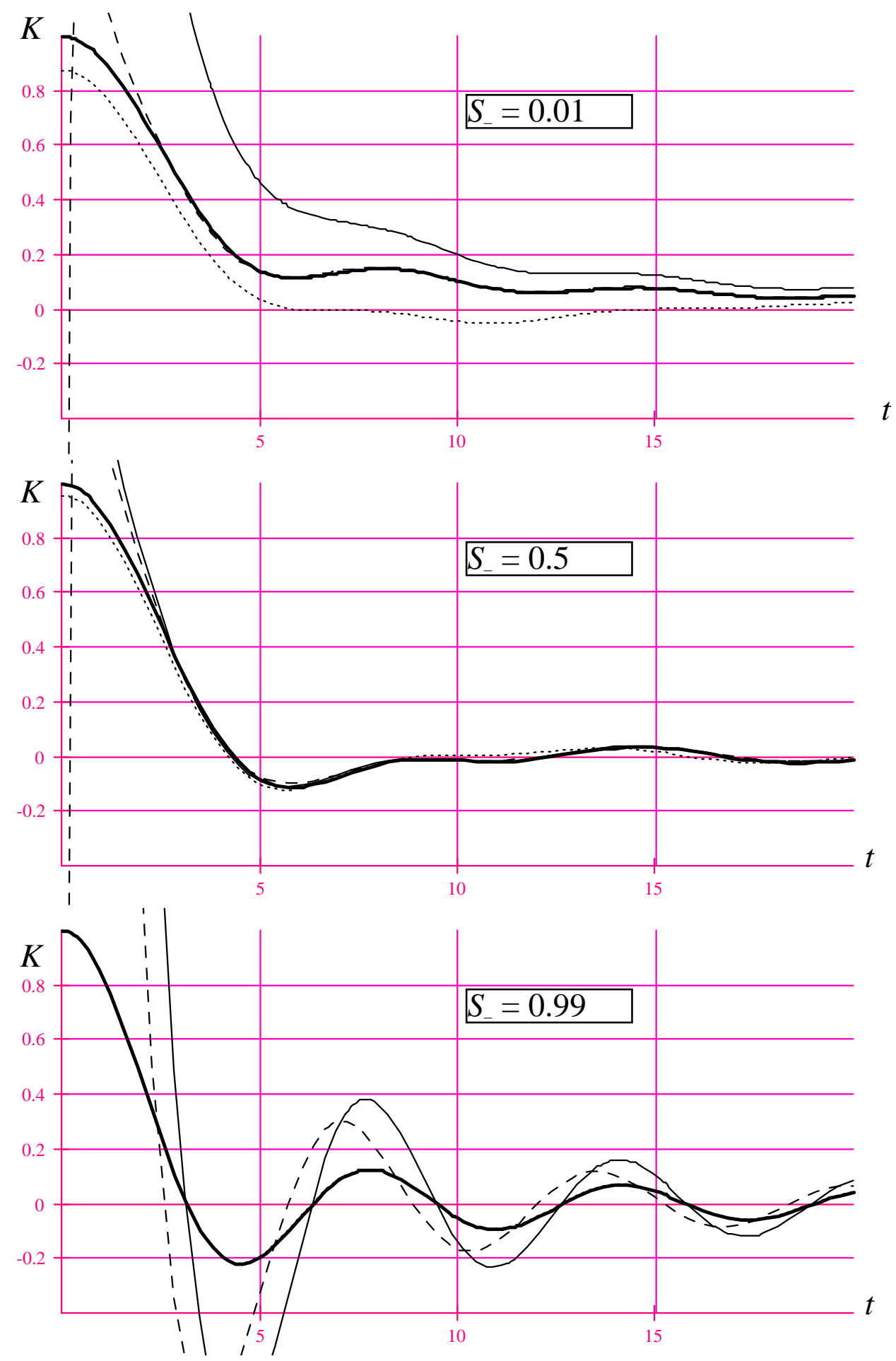

Figure 3: Kernel for $d=3, N=2$ as function of $t$ for $S_{+}=1$ and three values of $S_{-}$. Bold line: numerical evaluation of integral (36); thin line: general asymptotic form (42); dashed line: asymptotic form (45) for small $S_{-}$; dotted line: asymptotic form (43) for $S_{-} \approx S_{+}$. For $S_{-}=0.99 S_{+}$the error in the latter approximation is much smaller than the thickness of the lines. 